Sinergias educativas

ISSN: 2661-6661

compasacademico@icloud.com

Grupo Compás

Ecuador

\title{
La inteligencia emocional y su influencia en la formacio\#n de li\#deres estudiantiles de la Unidad Educativa Bilingu\#e Nueva semilla
}

Ruiz Galarza, Rita Rosario

La inteligencia emocional y su influencia en la formacio\#n de li\#deres estudiantiles de la Unidad Educativa

Bilingu\#e Nueva semilla

Sinergias educativas, vol. 2, núm. 1, 2017

Grupo Compás, Ecuador

Disponible en: http://www.redalyc.org/articulo.oa?id=573561688005

Esta obra está bajo una Licencia Creative Commons Atribución-NoComercial-SinDerivar 4.0 Internacional. 


\title{
La inteligencia emocional y su influencia en la formacio\#n de li\#deres estudiantiles de la Unidad Educativa Bilingu\#e Nueva semilla
}

\author{
Emotional intelligence and its influence on the formation of \\ student leaders of the Nueva seed Bilingual Educational Unit \\ Rita Rosario Ruiz Galarza ritaruizgalarza@Hotmail.com \\ Unidad Educativa Nueva Semilla, Ecuador \\ http://orcid.org/0000-0006-0322-457249
}

Sinergias educativas, vol. 2, núm. 1, 2017

Grupo Compás, Ecuador

Recepción: 15 Julio 2016

Aprobación: 25 Octubre 2016

Redalyc: http://www.redalyc.org/ articulo.oa?id $=573561688005$
Resumen: El presente trabajo tiene como objetivo descubrir la potencialidad de liderazgo en los estudiantes de la Unidad Educativa Bilingu\#e Nueva Semilla, a partir de la estimulacio\#n de la Inteligencia Emocional, logrando desarrollar habilidades cognitivas-psicomotora, las mismas que permitieron reconocer los tipos de liderazgo existentes, para de esta manera proporcionar las herramientas que permitan afianzar el problema de investigacio\#n. Para la realizacio\#n del mismo se utilizo\# una muestra de 30 estudiantes, se les aplico\# una encuesta incluyendo a los docentes y directivos a fin de proponer una gestio\#n metodolo\#gica para la estimulacio\#n de la inteligencia emocional y su incidencia en el desarrollo del liderazgo estudiantil. A partir de esta estrategia metodolo\#gica se lograra\# obtener nuevas perspectivas para el desarrollo de la calidad de vida, aumento de la autoestima, motivacio\#n y perfeccionamiento de la personalidad, logrando mayor sensibilidad hacia el entorno comunitario, desarrollando sus destrezas y habilidades o\#ptimas dentro del a\#mbito escolar.

Palabras clave: Inteligencia emocional, Liderazgo, Estrategias.

Abstract: The present work has as objective to discover the potential of leadership in the students of the Nueva Semilla Bilingual Educational Unit, from the stimulation of Emotional Intelligence, managing to develop cognitive-psychomotor skills, the same ones that allowed to recognize the types of existing leadership, in this way to provide the tools to strengthen the research problem. A sample of 30 students was used to carry it out, a survey was applied including teachers and managers in order to propose a methodological management for the stimulation of emotional intelligence and its impact on the development of student leadership. From this methodological strategy, new perspectives for the development of quality of life, increased self-esteem, motivation and improvement of personality will be achieved, 106 achieving greater sensitivity towards the community environment, developing their optimal skills and abilities within the school environment.

Keywords: Emotional intelligence, Leadership, Strategies.

\section{INTRODUCCIÓN}

El trabajo se desarrollo\# en la Unidad Educativa Bilingu\#e Nueva Semilla se pretende llevar a cabo un acercamiento al ana\#lisis y reflexio\#n de la inteligencia emocional en estudiantes del nivel secundario, donde se encuentran perfiles para liderar, pero sin una gui\#a que permita reconocer el tipo de liderazgo que esta\#n ejerciendo, puesto que hoy en di\#a este constructo ha tomado un papel protago\#nico para alcanzar el 
e\#xito, ante los inquebrantables escenarios que se les presenta en este mundo globalizado. Por tal razo\#n, los alumnos tendra\#n que estar mejor preparados para ejercer el liderazgo y ser capaces de enfrentar poseer una habilidad conductiva y pericia en la forma de plantear y resolver planes de accio\#n, aportando los mejores esfuerzos con la finalidad de encontrar los logros anhelados en las actividades escolares, puesto que una persona emocionalmente inteligente podra\# afrontar mejor los desafi\#os hoy en di\#a.

Esta propuesta, se estructura a partir de un marco teo\# rico acerca de la inteligencia emocional, su relacio\#n con el liderazgo, los beneficios que genera y los inconvenientes que se presentan por la carencia de la misma. Adema\#s de ser un apoyo se orienta sobre el complejo mundo que enfrentan los li\#deres estudiantiles, quienes dejan fluir con total libertad sus acciones y apasionamientos guiados por ese espi\# ritu juvenil que pretenden cambiar el mundo con sus suen\#os e ideales, muchos guiados por intereses personales que de cierta manera coartan verdaderas intenciones a favor de un grupo humano.

De tal manera que con este breve ana\#lisis se instaura el soporte conceptual que sustenta el planteamiento de la metodologi\#a de la investigacio\#n del estudio con un abordaje cualitativo, fundamentado en un trabajo investigativo el que se valoran los componentes integrantes de la inteligencia emocional, de acuerdo con Gadner, Goleman, Thorndike, quienes a trave\#s de sus aportes, permiten obtener las evidencias que demostrara\#n el planteamiento del problema propuesto.

Por tal razo\#n, los aciertos encontrados en esta investigacio\#n denotan la importancia de incluir a la inteligencia emocional como una herramienta de la gestio\#n educativa para la potencializacio\#n del liderazgo escolar, quienes se convertira\#n en los futuros actores que debera\#n desarrollar un liderazgo de excelencia sustentado en una visio\#n estrate\# gica e\#tica de compromiso social, para el logro de los objetivos mediante la construccio\#n de ventajas competitivas, para el siglo XXI.

\section{MATERIALES Y MÉTODOS}

El desarrollo del trabajo se efectu\#a en la Unidad Educativa Bilingu\#e Nueva Semilla, con la recopilacio\#n de informacio\#n en forma cualitativa, mediante la observacio\#n de los estudiantes, conjuntamente con la encuesta, entrevistas que determinara\# losresultadosyconclusionesalrespecto, asi\# mismo los me\#todos utilizados como son deductivo, inductivo, descriptivo. Al final los instrumentos servira\#n para la homogeneizacio\#n de los datos sacados de los estudiantes durante el proceso de investigacio\#n.

Durante muchos siglos el te\#rmino Inteligencia ha sido aplicado para describir las habilidades intelectuales de personas ingeniosas, astutas, obedientes, sumisas, adaptables, bien educadas; sin tomar en cuenta que el ser humano desde que nace, gracias a la inteligencia, se abre a la realidad, el conocimiento, al mundo, y personalizacio\#n de su conducta. 
En el a\#mbito educativo para muchos el concepto de inteligencia estaba ligado con el rendimiento, por lo cual un estudiante obteni\# a buenas calificaciones siendo considerado como un ser inteligente, Gadner la define como la capacidad de resolver problemas de la vida, o la habilidad de elaborar un producto $u$ ofrecer un servicio en un contexto cultural comunitario.

La inteligencia es una y mu\#ltiple a la vez porque es un sistema jerarquizado de procesos y estrategias cognitivas con niveles de interdependencia que corresponden a la herencia y organizacio\#n cerebral, que permite el desarrollo y evolucio\#n del ser humano, adema\#s funciona integrada al desarrollo de la personalidad y de sus emociones dependiendo de factores del mundo exterior como fi\#sico, social, cultural en el cual se desenvuelven.

Una de las habilidades ma\#s complicadas de desplegar y dominar con maestrii\#a es la regulacio\#n de nuestros estados emocionales, cuya habilidad es propicia para manejar o moderar nuestra reaccio\#n ante situaciones intensas positivas o negativas. En el contexto escolar, los educadores son los principales li\#deres emocionales de sus estudiantes.

En la de\#cada de los 80 hallamos dos desarrollos clave en este sentido: la Teori\#a de las Inteligencias Mu\#ltiples de Howard Gardner (1983) despue\#s modificada (Gardner, 1999) y la Teori\#a Tria\# rquica de Robert J. Sternberg (1985) cuyos planteamientos constituyen las teori\#as de la inteligencia emocional. La hipo\#tesis de Gardner (1983) representa un replanteamiento de las capacidades intelectuales, a la luz de una definicio\#n y de criterios fijados por el propio autor.

La propuesta de Sternberg, cuyo eje es estrictamente cognitivo, se fundamenta en aportaciones tanto acade\#micas como extra-acade\#micas, lo que le lleva a reconocer otras dimensiones de la inteligencia. Salvando las diferencias, ambos teo\# ricos coinciden en la necesidad de reformular el concepto de inteligencia, amplia\#ndolo y hacie\#ndolo compatible con los hallazgos de las neurociencias, los estudios transculturales, las aportaciones de las diferentes corrientes psicolo\#gicas e incluso las concepciones populares.

Acogie\#ndose en estas ideas, asi\# como en el reconocimiento de quienes las han formulado, autores como Salovey y Mayer o Goleman han desarrollado sus respectivas concepciones de la inteligencia emocional, con la conviccio\#n de estar recorriendo un camino iniciado desde la propia psicologi\#a acade\#mica.

Existe una discusio\#n entre los investigadores respecto si el liderazgo es considerado como un rasgo o un proceso. Mientras el enfoque de rasgo siguiere que los li\#deres tienen caracteri\#sticas y cualidades innatas que los distingue de las otras personas tal como lo manifiesta Northouse (2004) para otros se distingue como un proceso donde afirman que no so\#lo se trata de ciertas caracteri\#sticas sino un feno\#meno que depende i\#ntegramente del contexto en que se desenvuelven.

El enfoque funcional, ma\#s cercano, concibe al liderazgo en una situacio\#n como una propiedad de la estructura grupal, antes que como atributo individual. Como sen\#alan Cartwright y Zander (1960), en este 
enfoque se hallan impli\#citas dos ideas: primero que cualquier miembro de un grupo puede ser li\#der, en el sentido que puede encarar acciones que este\#n al servicio de las funciones grupales y segundo que muchas conductas diferentes adoptadas por distintas personas pueden cumplir una misma funcio\#n.

Por consiguiente el liderazgo exige el empleo de distintos recursos grupales en una actividad coordinada, lo cual significa que el li\#der ocupa un lugar como participante activo en el proceso. En el a\#mbito educativo, el liderazgo es un acontecimiento fa\#cil de observar, ya sea en su modalidad personal o colectiva. El surgimiento del conductor positivo puede ser fuente inagotable de provecho y recurso a utilizar en la interaccio\#n del proceso ensen\#anza- aprendizaje- Un alumno ejerciendo el liderazgo en este orden, va a contribuir como modelo de aprendizaje y ejemplo de comportamiento deseado.

La vigencia del liderazgo como fuerza impulsor permanece inalterable, y por consiguiente es necesario conocer co\#mo operan los li\#deres en te\#rminos de su capacidad para influir, persuadir y motivar a sus seguidores, De alguna forma se logra a trave\#s de sus actitudes y aptitudes, sobretodo de la percepcio\#n que tengan sus seguidores.

Las cualidades que identifican al li\#der deben basarse en la autoridad moral; es decir a trave\#s de una causa comu\#n, el poder del convencimiento con el resto de la comunidad y consigo mismo, de tal manera que el conocimiento y la habilidad para lograr sus objetivos lo lleve a la consecucio\#n del fin comu\#n.

Para ser un li\#der eficaz es fundamental no olvidar jama\#s que todos tenemos nuestro propio mundo, cuando alguien intenta comunicar algo, lo primero que hace es construirse un mapa mental de la idea o concepto. Es funcio\#n del li\#der reconocer los distintos estilos de pensamiento de sus colaboradores para proporcionarles el mayor nu\#mero de opciones y alternativas que encajen con dichos estilos.

La comprensio\#n puede ampliarse tanto utilizando los puntos fuertes de las personas como reforzando sus puntos de\#biles.Sialguiennoutilizanormalmente la visualizacio\#n, estimularle a pensar mediante ima\#genes puede resultarle u\#til. Si por el contrario visualiza bien, enfatizar enriquecer esta capacidad puede igualmente incrementar su comprensio\#n y su rendimiento en determinadas situaciones.

El paradigma humanista considera a los alumnos como entes individuales $\mathrm{u} \#$ nicos y diferentes de los dema\#s. Con iniciativa, necesidades personales de crecer, con potencialidad para desarrollar actividades y solucionar problemas creativamente. La finalidad del humanista no es gobernar sino formar a los estudiantes en la toma de decisiones en a\#mbitos en donde el respeto de los derechos de las personas, lo justo o injusto son cuestionables.

El humanismo (Herna\#ndez Rojas, 1998) se refiere al estudio y promocio\#n de los procesos integrales de la persona donde e\#sta es una organizacio\#n total que esta\# en continuo proceso de desarrollo y la persona debe ser estudiada en su contexto interpersonal y social. 
Las conductas humanas no son fragmentarias e implican aspectos naturales como el egoi\#smo, el amor, las relaciones interpersonales afectivas, las cuestiones e\#ticas, los valores como la bondad o aspectos naturales fi\#sicos como la muerte, la sexualidad, entre muchos otros.

Por lo tanto desde el punto de vista humanista, la educacio\#n se debe centrar en ayudar a los alumnos para que decidan lo que son y lo que quieren llegar a ser. Tienen la idea de que los alumnos son diferentes y les ayuda a ser ma\#s como ellos y menos como los dema\#s.

\section{CONCLUSIONES}

A pesar de todos los problemas y limitaciones, la educacio\#n de nuestro pai\#s busca nuevos caminos, inicia constantes cambios, se arriesga a nuevas aventuras, pero este tiempo de bu\#squeda, es lo que lleva a consolidar el tema de investigacio\#n, no sin antes afirmar que dentro de la institucio\#n es casi nulo un buen liderazgo estudiantil. Por lo tanto plantear ciertas estrategias que estimulen y permitan optimizar la inteligencia emocional para obtener un aute\#ntico liderazgo es lo que llevara\# alcanzar el objetivo propuesto.

El liderazgo estudiantil que se propone es ser capaz de promover una adecuada comunicacio\#n entre sus integrantes, ser tolerantes ante las diversas circunstancias que se pueden presentar, ya que esto demostrari\#a el buen manejo de sus emociones, cuya caracteri\#stica lo considerari\#a como un aute\#ntico li\#der, ya que al dirigir a un grupo humano debe ser capaz de inculcar valores; humanos, sociales y e\#ticos, que les permita actuar de manera diferente tanto en el a\#mbito escolar como social.

Cabe recalcar que la inteligencia emocional es el motor principal que permite al gui\#a, conductor, a mover las piezas claves para demostrar su verdadero dominio, justo y sensato de su equipo en un liderazgo democra\#tico, que busca el desarrollo permanente de sus compan\#eros, ya que el dominio de sus emociones es un verdadero e\#xito en las funciones que logre desempen\#ar.

Por lo tanto lo que se busca es promover y fortalecer el liderazgo a trave\#s de la inteligencia emocional, a fin de que muchos puedan reconocer la capacidad de liderazgo que poseen y desempen\#ar con calidad y calidez esta funcio\#n tan importante en nuestra e\#poca.

Pero sera\# realmente la escuela un espacio o\#ptimo para desarrollar un perfil de liderazgo bajo estas caracteri\#sticas, o sera\# ma\#s una cuestio\#n innata?

\section{Referencias}

LINGREN,C. (1978). Introducción a la Psicología social. México: Cubierta Deslucida

Mora Me\#rida, J.A. (2007). efectos sobre la intensidad de los recuerdos escolares. Anales de Psicología. 31(3), 1035-1043

FERNA\#NDEZ, P., \& EXTREMERA, N. (2005). Inteligencia emocional percibida y diferencias individuales en la meta-conocimiento de los estados 
emocionales: una revisión de los estudios con el TMMS. Ansiedad y Estrés. 10(2-3), 287-298

Isaza Zapata, G.M., \& Calle Piedrahi\#ta, J. S. (2016). Un acercamiento a la comprensión del perfil de la Inteligencia Emocional. Revista Latinoamericana de Ciencias Sociales, Niñez y Juventud. 14(1), 331-345

Ferna\#ndez Marti\#nez, A.M. \& Montero Garci\#a, I. (2016). Aportes para la educación de la Inteligencia Emocional desde la Educación Infantil. RLCSNJ. 14(1), 53-66

Del Pino Pen\#a, R. \& Aguilar Ferna\#ndez, M.A. (2013). La inteligencia emocional como una herramienta de la gestión educativa para el liderazgo estudiantil. Cuadernos de Administración. 29(50), 132-141

Santamari\#a, C., \& Sa\#nchez, M.A. (2011). Participación y liderazgo estudiantil: Una apuesta por la ciudadanía activa. Colombia Médica. 42(2), 103-112

Fragoso Luzuriaga, R. (2015). Inteligencia emocional y competencias emocionales en educación superior, ¿un mismo concepto?. Revista Iberoamericana de Educación Superior. 6(16), 110-125.

Ferragut, M. \& Fierro,A. (2012). Inteligencia emocional, bienestar personal $y$ rendimiento académico en preadolescentes. Estados Unidos: AI2. Recuperado de https://www.semanticscholar.org/paper/Inteligenciaemocional\%2C-bienestar-personal-y-en-Ferragut-

Fierro/410a7b369b7d858dbb96c16c2329f9670a580104

ROJAS, M. (1998). Observando Observaciones de una Modernidad ¿Inacabada?. Universidad de Chile. Chile 